\title{
Equivalência Semântica da versão em português do "Body Change Inventory"
}

\author{
Semantic Equivalence of the Brazilian Portuguese version \\ of the "Body Change Inventory"
}

\author{
Maria Aparecida Conti ${ }^{1}$ \\ Maria Elisa Caputo Ferreira ${ }^{2}$ \\ Ana Carolina Soares Amaral ${ }^{2}$ \\ Norman Hearst ${ }^{3}$ \\ Táki Athanássios Cordás ${ }^{4}$ \\ Fernanda Baeza Scagliusi ${ }^{5}$
}

${ }^{1}$ Núcleo de estudo e atendimento psicológico (NEAP), Universidade Cruzeiro do Sul. R.

Taiuvinha 26, São Miguel Paulista. 08060-070 São

Paulo SP.maconti@usp.br

${ }^{2}$ Laboratório de Estudos do

Corpo, Faculdade de

Educação Física e Desportos,

Universidade Federal de Juiz

de Fora.

${ }^{3}$ Universidade da

Califórnia.

${ }^{4}$ Programa de Transtornos

Alimentares, Instituto de

Psiquiatria, Universidade de São Paulo.

${ }^{5}$ Campus Baixada Santista, Depto de Ciências da Saúde, Universidade Federal de São Paulo

\begin{abstract}
With the increase in research on the components of Body Image, validated instruments are needed to evaluate its dimensions. The Body Change Inventory (BCI) assesses strategies used to alter body size among adolescents. The scope of this study was to describe the translation and evaluation for semantic equivalence of the BCI in the Portuguese language. The process involved the steps of (1) translation of the questionnaire to the Portuguese language; (2) back-translation to English; (3) evaluation of semantic equivalence; and (4) assessment of comprehension by professional experts and the target population. The six subscales of the instrument were translated into the Portuguese language. Language adaptations were made to render the instrument suitable for the Brazilian reality. The questions were interpreted as easily understandable by both experts and young people. The Body Change Inventory has been translated and adapted into Portuguese. Evaluation of the operational, measurement and functional equivalence are still needed.
\end{abstract}

Key words Body Image, Validation studies, Translation (process), Translation (product)
Resumo Com o crescimento das pesquisas acerca dos componentes da imagem corporal, fazem-se necessários instrumentos válidos que avaliem suas dimensões. O Body Change Inventory (BCI) avalia as estratégias adotadas para modificação do corpo entre adolescentes. O objetivo deste estudo foi descrever o processo de tradução e avaliação da equivalência semântica do BCI para a língua portuguesa. O processo envolveu as etapas de (1) tradução do questionário para o idioma português; (2) retrotradução para o inglês; (3) avaliação da equivalência semântica; e, (4) análise da compreensão verbal por especialistas e jovens brasileiros. As seis subescalas que compõem o instrumento foram traduzidas para o português. Foram realizadas adaptações na linguagem a fim de tornar o instrumento adequado à realidade brasileira. As questões foram interpretadas como de fácil compreensão tanto por especialistas quanto pelos jovens. O Questionário de Mudança Corporal encontra-se traduzido e adaptado para o idioma português, sendo ainda necessária a avaliação das equivalências operacional, de mensuração e funcional.

Palavras-chave Imagem corporal, Estudos de validação, Tradução (processo), tradução (produto) 


\section{Introdução}

A insatisfação corporal está presente na vivência de adolescentes de ambos os sexos. Entre as meninas, o desejo de possuir um corpo mais magro foi o principal foco das pesquisas durante vários anos $^{1}$. Com o crescimento das investigações acerca da insatisfação corporal masculina, verificouse que os homens também se apresentam preocupados com o próprio corpo; porém, tudo indica que essa insatisfação está relacionada à muscularidade e não apenas ao corpo magro ${ }^{2,3}$.

A crescente prevalência de insatisfação entre meninos e meninas pode se justificar pela influência direta de diversos fatores, tais como idade, sexo e maturação sexual ${ }^{4}$, além das influências socioculturais da mídia, dos pais e dos amigos, sendo estas especialmente relacionadas aos ideais de aparência.

Como resultado da internalização das mensagens acerca dos ideais de corpo pregados nas sociedades pós-modernas, os adolescentes têm desenvolvido altos graus de insatisfação corporal quando percebem que seus corpos não estão de acordo com os padrões estabelecidos 5 . A partir disso, eles adotam estratégias para que seus corpos se aproximem do modelo desejado. Tanto para meninos quanto para meninas, as estratégias de mudança corporal utilizadas vão desde condutas alimentares até a realização de exercícios físicos e/ ou utilização de suplementação alimentar ${ }^{6}$.

Estudos revelam que, entre os meninos, as estratégias mais comumente utilizadas para a modificação do corpo são exercícios para aumentar a massa muscular ${ }^{7,8}$ e uso de anabolizantes ${ }^{9}$. Entre as meninas, as técnicas mais utilizadas estão relacionadas à perda de peso, como dietas restritivas e a prática de atividades físicas ${ }^{1}$.

Muitas vezes, entretanto, a adoção de certos comportamentos se torna prejudicial à saúde, como no caso da utilização de esteróides anabolizantes, da prática excessiva de exercícios físicos e do desenvolvimento de transtornos alimentares $^{6}$, fazendo-se necessárias, cada vez mais, investigações acerca das principais estratégias de modificação do corpo ${ }^{9,10}$.

A preocupação com os aspectos precursores e os efeitos dessas estratégias de mudança corporal têm levado vários pesquisadores a investigarem o papel destes comportamentos em diversas populações ${ }^{2,11-13}$. Para tanto, são necessários instrumentos válidos na avaliação dos componentes da imagem corporal ${ }^{14}$.

As estratégias de mudança do corpo podem ser avaliadas por instrumentos específicos, como o Body Change Inventory, desenvolvido por Ricciardelli e $\mathrm{McCabe}^{1}$ para a população adolescente, de ambos os sexos. Esse questionário é composto por seis sub-escalas que avaliam: práticas alimentares (9 itens), uso de suplementos alimentares ( 9 itens) e as estratégias adotadas para perder ( 9 itens) ou aumentar o peso ( 9 itens), aumentar o tônus (6 itens) e o tamanho muscular (6 itens). As respostas são dadas em uma escala Likert de pontos, variando de 1 (always) a 5 (never). As subescalas demonstraram altos valores de consistência interna $(r>0,92)$ e de estabilidade por meio do teste-reteste $(r>0,75)^{1}$. Além disso, as autoras destacam a importância deste instrumento, já que avalia preocupações com a imagem corporal presentes também entre os meninos (como estratégias para aumento do tônus muscular e uso de suplementos), não focando apenas nos comportamentos tipicamente femininos (como perda de peso).

Este instrumento tem sido muito utilizado em pesquisas que buscam investigar as estratégias de mudança corporal ${ }^{15-17}$, mas não existem ainda registros de sua validade para amostras brasileiras. Por ser autoaplicável, o Body Change Inventory é de fácil utilização em grandes populações, além de possibilitar o acesso aos comportamentos para a modificação do corpo, quer no espaço clínico como também no epidemiológico.

A partir disso, este trabalho objetiva descrever as etapas iniciais de seu processo de adaptação transcultural, que integra a tradução, retrotradução, análise da equivalência semântica e compreensão verbal.

\section{Métodos}

As etapas do processo de adaptação transcultural basearam-se nos procedimentos sugeridos por Reichenheim e Moraes ${ }^{18}$ e aplicados por Conti et al. ${ }^{19}$ e Amaral et al. ${ }^{20}$.

O instrumento foi traduzido da versão original (inglês) para a língua portuguesa e na sequência recebeu uma retrotradução para a língua inglesa. Foi realizada uma revisão técnica do instrumento e avaliada a equivalência semântica entre o questionário original e as versões traduzida e retrotraduzida, com a proposição de uma versão (V1). Em seguida, a V1 passou por avaliações de especialistas e estudantes e ao término desta etapa, uma nova versão foi proposta (V2).

A primeira etapa consistiu na tradução do instrumento original do idioma inglês para o português, feita por um pesquisador experiente 
e fluente em inglês (MC). Para a segunda etapa, a versão (tradução) foi novamente traduzida para o inglês, por um professor nativo de língua inglesa $(\mathrm{NH})$. A etapa seguinte referiu-se à revisão técnica e à avaliação da equivalência semântica entre o instrumento original e as versões produzidas pelos tradutores, desenvolvida por dois profissionais (um psicólogo e um nutricionista) especialistas na área de adaptação de escala. $\mathrm{Na}$ avaliação da equivalência semântica dois aspectos foram priorizados: o significado referencial (denotativo) e o significado geral (conotativo), na avaliação da versão traduzida (português) em relação à versão final (inglês), com o intuito de se garantir a transferência dos significados das palavras entre os dois idiomas ${ }^{18}$. Foram realizados ajustes e uma versão adaptada foi proposta (V1).

$\mathrm{Na}$ terceira etapa, para avaliar a clareza e o grau de compreensão de cada item e do instrumento com um todo, a versão adaptada (V1) do instrumento foi apresentada a 9 profissionais especialistas da área de Transtornos Alimentares, com experiência clínica superior a 4 anos (três psicólogos, três nutricionistas, um educador físico, um enfermeiro, um psiquiatra). Foi solicitado a cada profissional que lesse atentamente cada item e respondessem uma escala verbalnumérica adaptada ${ }^{19,20}$ de acordo com a seguinte questão: "Você entendeu o que foi perguntado?". As respostas eram do tipo escala Likert: 0 - não entendi nada; 1 - entendi só um pouco; 2 - entendi mais ou menos; 3 - entendi quase tudo, mas tive algumas dúvidas; 4 - entendi quase tudo; 5entendi perfeitamente e não tenho dúvidas. Conforme Conti et al. ${ }^{21}$ foi estabelecido que as respostas $0,1,2$ e 3 seriam consideradas como indicadores de uma compreensão insuficiente. Foi solicitado ainda, caso o especialista não compreendesse a questão ou a linguagem não parecesse adequada que sugerisse alterações, justificando os motivos.

Esta mesma escala foi apresentada a 47 jovens, sendo 20 mulheres (21,2 anos e desvio padrão de 1,9 anos) e 27 homens (22,7 anos e desvio padrão de 2,3 anos), estudantes do curso de Educação Física de uma instituição pública de Ensino Superior. Os jovens foram selecionados de forma aleatória simples e concordaram em participar voluntariamente. $\mathrm{O}$ instrumento foi aplicado na apresentação de uma escala verbalnumérica adaptada ${ }^{19}$, exatamente da mesma forma como aplicada aos especialistas, seguindo os procedimentos metodológicos descritos anteriormente. Almejou-se avaliar o grau de compreensão de cada questão e do instrumento em sua íntegra. Ao término do preenchimento da escala foi solicitado a este jovem que registrasse suas sugestões, caso percebesse alguma dificuldade para a compreensão nos itens que compõem o instrumento. Uma nova versão do instrumento foi formulada a partir destes dados (V2).

Para as análises estatísticas utilizou-se o programa SPSS versão 16.0, a fim de desenvolver uma análise descritiva dos dados (média, desvios-padrão, valores mínimos e máximos, frequências).

O presente estudo está de acordo com as normas no 196 de 10/10/1996 22 do Conselho Nacional de Saúde e foi aprovado pelo Comitê de Ética e Pesquisa do Hospital das Clínicas da Faculdade de Medicina da Universidade de São Paulo.

\section{Resultados}

\section{Tradução e Equivalência Semântica}

Na tradução do instrumento houve necessidade de adaptação de inúmeros termos e expressões. Na orientação para o preenchimento do instrumento, na versão original, o tempo verbal está no presente is designed, mas para o nosso idioma foi adaptado para o tempo passado, ficando como "foi preparado", exatamente por se adequar aos nossos padrões de comunicação.

Sinônimos foram observados na retrotradução, como por exemplo: was proposed por is designed; just circle por simply circle; we only want por we just want, o que de fato confirmou que o significado de cada expressão foi mantido na tradução do instrumento.

Na primeira escala - "Hábitos alimentares", um sinônimo foi identificado na retrotradução: large para a lot (item 1), sendo mantido a palavra "grande". Outro aspecto relevante referiu-se à concordância nominal, nos itens 2, 5 e 8, respectivamente. A expressão stuffing yourself foi retrotraduzida para until you feel stuffed; do original find that all you can think about is food para it seem that food is all you can think about e like stuffing yourself with food para "desire to binge with food. Desta forma, na versão 1 (V1) garantiu-se a preservação do significado semântico de cada item.

Na escala 2 - "Suplementos Alimentares", os sinônimos foram observados, a começar pelo próprio título da escala: food para dieting. $\mathrm{Na}$ expressão (item 2) feel like para feel the desire. Um aspecto relevante a ser destacado diz respeito à expressão food supplements, pois para o idioma 
inglês engloba além do alimento, remédios e complementos alimentares, o que não acontece com o idioma português. Assim, para essa escala, a tradução desta expressão ficou como "substância”. Adaptações foram realizadas em cada item, pois ao descrever o exemplo para food supplements - "substância", repetia-se expressões. Isto pode ser observado nos itens 1 a 4 . No item 1 , cita-se como exemplo diets pills, que para o nosso idioma tem o significado de "remédio para emagrecer". Assim, a expressão final deste item, após a citação do exemplo "para emagrecer" foi excluída, pois a própria citação "remédio para emagrecer" esclarece o conteúdo do item. Este mesmo cuidado foi adotado nos itens 2 a 4 .

Interessante observar que para essa escala, o mesmo conteúdo é avaliado nos itens 2, 3, 4 e 5, somente com a distinção dos aspectos afetivos (sente), cognitivo (pensa e preocupa-se) e comportamental (usa). Esta característica se repete ao longo do questionário.

Para a escala 3 - "Estratégias de mudança corporal para perder peso", novamente conteúdos semelhantes foram observados, como nos itens 4 e 6, com a expressão doing more exercise sendo retrotraduzida para exercise more. Já para os itens 7, 8 e 9 a expressão food supplements por dieting supplements sendo preservado a expressão suplementos alimentares.

$\mathrm{Na}$ escala 4 - "Estratégias de mudança corporal para aumentar peso", o sinônimo increase na tradução e gain, na retrotradução pode ser observado no próprio título da escala. Duas expressões foram identificadas, sendo a primeira put on weight por gain weight e novamente food supplements, que foi adequada para "suplemento alimentar".

$\mathrm{Na}$ escala 5 - "Estratégias de mudança corporal para aumento do tônus muscular" e escala 6 - "Estratégias de mudança corporal para aumento do tamanho muscular" algumas observações podem ser realizadas. Para a escala 5, somente um sinônimo foi identificado em quase todos os itens, do original to increase para improve. E já na escala 6, a expressão to make your muscle bigger para to get bigger.

As etapas de tradução, retrotradução e a primeira versão (V1) podem ser observadas no Quadro 1.

Quadro 1. Avaliação da Equivalência semântica do Body Change Inventory. São Paulo, 2008.

\begin{tabular}{|c|c|}
\hline Documento original & Versão traduzida \\
\hline Body Change Questionnaire & Questionário de Mudança Corporal \\
\hline Eating Practices & Hábitos Alimentares \\
\hline 1. How often do you quickly eat a large amount of food? & $\begin{array}{l}\text { 1. Com que frequência você come uma grande quantidade } \\
\text { de comida? }\end{array}$ \\
\hline 2. How often do you eat to the point of stuffing yourself? & 2. Com que frequência você come ao ponto de se estufar? \\
\hline $\begin{array}{l}\text { 3. How often do you eat a lot of food when you're not even } \\
\text { hungry? }\end{array}$ & $\begin{array}{l}\text { 3. Com que frequência você come uma grande quantidade } \\
\text { de comida quando você nem está com fome? }\end{array}$ \\
\hline 4. How often do you experience urges to eat and eat? & $\begin{array}{l}\text { 4. Com que frequência você vivencia um impulso para } \\
\text { comer e comer? }\end{array}$ \\
\hline $\begin{array}{l}\text { 5. How often do you find that all you can think about is } \\
\text { food? }\end{array}$ & $\begin{array}{l}\text { 5. Com que frequência você acha que tudo o que você } \\
\text { consegue pensar é sobre comida? }\end{array}$ \\
\hline $\begin{array}{l}\text { 6. How often do you think about eating a large amount of } \\
\text { food? }\end{array}$ & $\begin{array}{l}\text { 6. Com que frequência você pensa em comer uma grande } \\
\text { quantidade de comida? }\end{array}$ \\
\hline $\begin{array}{l}\text { 7. How often do you think about food when you're not } \\
\text { even hungry? }\end{array}$ & $\begin{array}{l}\text { 7. Com que frequência você pensa sobre comida quando } \\
\text { você nem está com fome? }\end{array}$ \\
\hline 8. How often do you feel like stuffing yourself with food? & $\begin{array}{l}\text { 8. Com que frequência você sente vontade de se } \\
\text { empanturrar com a comida? }\end{array}$ \\
\hline 9. How often do you eat a lot when feeling anxious? & $\begin{array}{l}\text { 9. Com que frequência você come muito quando sente-se } \\
\text { ansioso? }\end{array}$ \\
\hline
\end{tabular}




\section{Compreensão Verbal}

Para os especialistas e a população alvo as questões demonstraram ser de fácil compreensão, registrando-se valor médio superior a 3,9 (valor máximo $=5,0$ e 4,9, respectivamente).

Os estudantes apontaram para a fato de as perguntas serem semelhantes e repetitivas. Além disso, a expressão "comer e comer", da questão 5 da subescala Hábitos Alimentares foi apontada como confusa por vários participantes.

A partir destes resultados e com base nas sugestões dadas por especialistas e estudantes, foi produzida a versão final do Questionário de Mudança Corporal, na qual agrupou-se o início das perguntas ("Com que frequência”), evitando a repetição, e alterou-se as expressões que causaram dúvidas (Quadro 2).

\section{Discussão}

Embora haja inúmeras estratégias para o processo da adaptação cultural, que vão desde a simples tradução por parte dos pesquisadores ao processo mais minucioso que abrange e enfatiza a necessidade de diferentes nuanças na adaptação cultural ${ }^{14}$, estudos recentes comprovam a importância em se valorizar as etapas de operacionalização na adaptação do instrumento ${ }^{18}$. Desta forma garante-se a veracidade e a qualidade da informação coletada. O presente estudo cumpre este fim, apresentando uma escala e descrevendo seu processo de tradução e de avaliação de sua equivalência semântica.

A fim de preservar o sentido do enunciado original na tradução para a língua portuguesa, algumas palavras foram alteradas. Porém, após o processo de retrotradução, verificou-se que o significado foi mantido, sendo que muitas palavras foram substituídas por sinônimos nesta etapa. A alteração de palavras do instrumento a fim de adequá-lo ao idioma alvo da tradução está de acordo com o que propõem Widelfelt et al. ${ }^{23}$, para os quais os enunciados devem ser avaliados criticamente a fim de identificar quais itens não podem ser traduzidos de forma literal. Os autores afirmam que este processo garante que a intencionalidade do instrumento original seja preservada.

Corroborando estes pressupostos, Jorge J $^{24}$ afirma que significado geral transcende a literalidade dos termos, sendo necessário considerar os

Quadro 1. continuação

\begin{tabular}{|c|c|}
\hline Versão Retrotraduzida & Versão Final (V1) \\
\hline Questionnaire About Body Changes & Questionário de Mudança Corporal \\
\hline Eating Habits & Hábitos Alimentares \\
\hline 1. How often do you quickly eat a large amount of food? & 1. Com que frequência você come uma grande quantidade \\
\hline 2. How often do you eat until you feel stuffed? & 2. Com que frequência você come ao ponto de se estufar? \\
\hline $\begin{array}{l}\text { 3. How often do you eat a lot of food when you're not even } \\
\text { hungry? }\end{array}$ & $\begin{array}{l}\text { 3. Com que frequência você come uma grande quantidade } \\
\text { de comida quando você nem está com fome? }\end{array}$ \\
\hline 4. How often do you have an impulse to eat and eat? & $\begin{array}{l}\text { 4. Com que frequência você vivencia um impulso para } \\
\text { comer e comer? }\end{array}$ \\
\hline $\begin{array}{l}\text { 5. How often does it seem that food is all you can think } \\
\text { about? }\end{array}$ & 5. Com que frequência você só pensa em comida? \\
\hline 6. How often do you think about eating a lot of food? & $\begin{array}{l}\text { 6. Com que frequência você pensa em comer uma grande } \\
\text { quantidade de comida? }\end{array}$ \\
\hline $\begin{array}{l}\text { 7. How often do you think about food when you're not even } \\
\text { hungry? }\end{array}$ & $\begin{array}{l}\text { 7. Com que frequência você pensa sobre comida quando } \\
\text { você nem está com fome? }\end{array}$ \\
\hline 8. How often do you feel a desire to binge with food? & $\begin{array}{l}\text { 8. Com que frequência você sente vontade de comer até se } \\
\text { empanturrar? }\end{array}$ \\
\hline 9. How often do you eat a lot when you're anxious? & $\begin{array}{l}\text { 9. Com que frequência você come muito quando se sente } \\
\text { ansioso? }\end{array}$ \\
\hline
\end{tabular}


aspectos culturais da população alvo. Este cuidado foi tomado no presente estudo. Ainda foram necessárias adaptações com inserções de ex- pressões, padronização de pronomes e tempos verbais, com o intuito de garantir a compreensão do conteúdo expresso na escala original. Sen-

Quadro 1. continuação

\begin{tabular}{|c|c|}
\hline Documento original & Versão traduzida \\
\hline Body Change Questionnaire & Questionário de Mudança Corporal \\
\hline Food Supplements & Suplementos Alimentares \\
\hline
\end{tabular}

1. How often do you take food supplements (for example, diet pills) to lose weight?

2. How often do you feellike taking food supplements (for example, diet pills) to lose weight?

3. How often do you think about taking food supplements (for example, diet pills) to lose weight?

4. How often do you worry about taking food supplements (for example, diet pills) to lose weight?

5. How often do you take food supplements (for example, Sustagen) to increase your muscles?

6. How often do you feel like taking food supplements (for example, Sustagen) to increase your muscles?

7. How often do you think about taking food supplements (for example, Sustagen) to increase your muscles?

8. How often do you take steroids?

9. How often do you think about taking steroids?
1. Com que frequência você usa substâncias para perder peso (por exemplo, remédios para emagrecer) para emagrecer?

2. Com que frequência você sente vontade de usar substâncias para perder peso (por exemplo, remédios para emagrecer) para emagrecer?

3. Com que frequência você pensa em usar substâncias para perder peso (por exemplo, remédios para emagrecer) para emagrecer?

4. Com que frequência você se preocupa em usar substâncias para perder peso (por exemplo, remédios para emagrecer) para emagrecer?

5. Com que frequência você usa suplementos alimentares (por exemplo, Sustagen) para aumentar seus músculos? 6. Com que frequência você sente vontade de tomar suplementos alimentares (por exemplo, Sustagen) para aumentar seus músculos?

7. Com que frequência você pensa em tomar suplementos alimentares (por exemplo, Sustagen) para aumentar seus músculos?

8. Com que frequência você usa esteroides-anabolizantes?

9. Com que frequência você pensa em usar esteroidesanabolizantes?

\section{Estratégias de mudança corporal para perder peso}

1. How often do you think about eating less so that you can lose weight?

2. How often do you eat less to lose weight?

3. How often do worry about eating less to lose weight?

4. How often do you think about doing more exercise to lose weight?

5. How often do you do more exercise to lose weight?

6. How often do you worry about doing more exercise to lose weight?

7. How often do you change the amount of food supplements you use to lose weight?

8. How often do you think about changing the amount of food supplements you use to lose weight?

9. How often do you worry about changing the amount of your food supplements you use to lose weight?
1. Com que frequência você pensa em comer menos para que você possa perder peso?

2. Com que frequência você come menos para perder peso?

3. Com que frequência você se preocupa em comer menos para perder peso?

4. Com que frequência você pensa em fazer mais exercícios para perder peso?

5. Com que frequência você faz mais exercícios para perder peso?

6. Com que frequência você se preocupa em fazer mais exercícios para perder peso?

7. Com que frequência você muda a quantidade de suplementos alimentares que você usa para perder peso? 8. Com que frequência você pensa em mudar a quantidade de suplementos alimentares que você usa para perder peso? 9. Com que frequência você se preocupa em mudar a quantidade de suplementos alimentares que você usa para perder peso? 
do assim, zelou-se pela pertinência, adequação e aceitabilidade do estilo empregado em cada item analisado.
Assim, a estrutura geral da escala foi preservada, com a manutenção de todos os itens que compõem o instrumento original.

Quadro 1. continuação

\begin{tabular}{|c|c|}
\hline Versão Retrotraduzida & Versão Final (V1) \\
\hline Questionnaire About Body Changes & Questionário de Mudança Corporal \\
\hline Dieting Supplements & Suplementos Alimentares \\
\hline
\end{tabular}

1. How often do you use substances (for example, diet pills) to lose weight?

2. How often do you feel the desire to use substances (for example, diet pills) to lose weight?

3. How often do you think about using substances (for example, diet pills) to lose weight?

4. How often do you worry about taking food supplements (for example, diet pills) to lose weight?

5. How often do you use dietary supplements (for example, Sustagen) to make your muscles bigger?

6. How often do you feel the desire to use dietary supplements (for example, Sustagen) to make your muscles bigger?

7. How often do you think the desire to use dietary supplements (for example, Sustagen) to make your muscles bigger?

8. How often do you use anabolic steroids?

9. How often do you think about using anabolic steroids?
1. Com que frequência você usa substâncias para perder peso, por exemplo, remédios para emagrecer?

2. Com que frequência você sente vontade de usar substâncias para perder peso, por exemplo, remédios para emagrecer?

3. Com que frequência você pensa em usar substâncias para perder peso, por exemplo, remédios para emagrecer?

4. Com que frequência você se preocupa em usar substâncias para perder peso, por exemplo, remédios para emagrecer?

5. Com que frequência você usa suplementos alimentares (por exemplo, Sustagen) para aumentar seus músculos? 6. Com que frequência você sente vontade de tomar suplementos alimentares (por exemplo, Sustagen) para aumentar seus músculos?

7. Com que frequência você pensa em tomar suplementos alimentares (por exemplo, Sustagen) para aumentar seus músculos?

8. Com que frequência você usa esteroides-anabolizantes?

9. Com que frequência você pensa em usar esteroidesanabolizantes?

\section{Estratégias de mudança corporal para perder peso}

1. How often do you think about eating less so that you can lose weight?

2. How often do you eat less to lose weight?

3. How often do worry about eating less to lose weight?

4. How often do you think about exercising more to lose weight?

5. How often do you do exercise more to lose weight?

6. How often do you worry about exercising more to lose weight?

7. How often do you change the amount of dieting supplements you use to lose weight?

8. How often do you think about changing the amount of dieting supplements you use to lose weight?

9. How often do you worry about changing the amount of your dieting supplements you use to lose weight?
1. Com que frequência você pensa em comer menos para perder peso?

2. Com que frequência você come menos para perder peso?

3. Com que frequência você se preocupa em comer menos para perder peso?

4. Com que frequência você pensa em fazer mais exercícios para perder peso?

5. Com que frequência você faz mais exercícios para perder peso?

6. Com que frequência você se preocupa em fazer mais exercícios para perder peso?

7. Com que frequência você muda a quantidade de suplementos alimentares que você usa para perder peso? 8. Com que frequência você pensa em mudar a quantidade de suplementos alimentares que você usa para perder peso? 9. Com que frequência você se preocupa em mudar a quantidade de suplementos alimentares que você usa para perder peso? 


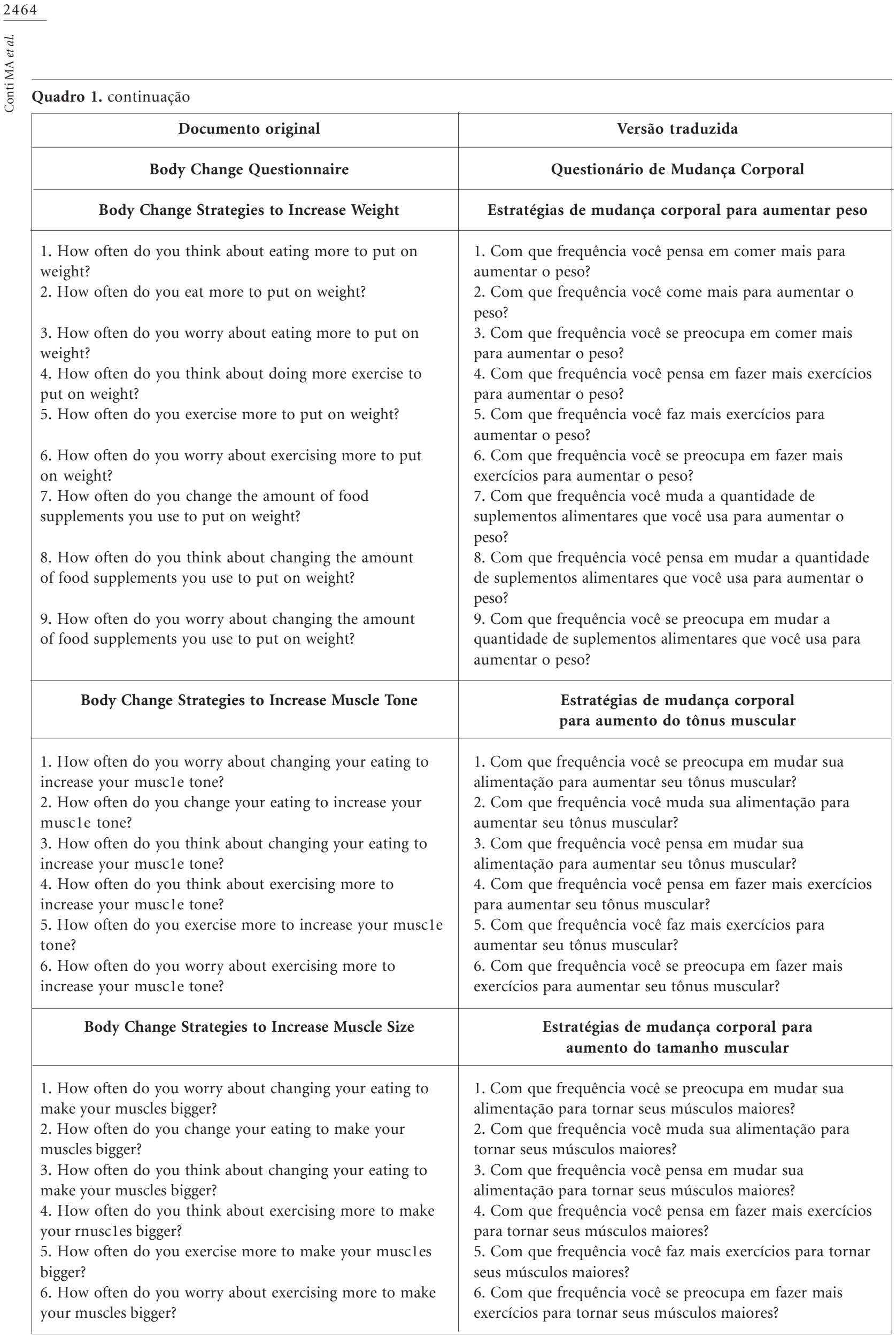


Quadro 1. continuação

\begin{tabular}{|c|c|}
\hline Versão Retrotraduzida & Versão Final (V1) \\
\hline Questionnaire About Body Changes & Questionário de Mudança Corporal \\
\hline Strategies for Body Change to Gain Weight & Estratégias de mudança corporal para Aumento de Peso \\
\hline 1. How often do you think about eating more to gain weight? & $\begin{array}{l}\text { 1. Com que frequência você pensa em comer mais para } \\
\text { aumentar seu peso? }\end{array}$ \\
\hline
\end{tabular}

2. How often do you eat more to gain weight?

2. Com que frequência você come mais para aumentar seu peso?

3. How often do you worry about eating more to gain weight?

3. Com que frequência você se preocupa em comer mais para aumentar o peso?

4. How often do you think about exercising more to gain weight?

4. Com que frequência você se pensa em fazer mais exercícios para aumentar seu peso?

5. How often do you exercise more to gain weight?

5. Com que frequência você faz mais exercícios para aumentar seu peso?

6. How often do you worry about exercising more to gain weight?

6. Com que frequência você se preocupa em fazer mais exercícios para aumentar o peso?

7. How often do you change the amount of dieting supplements you use to gain weight?

7. Com que frequência você muda a quantidade de suplementos alimentares que você usa para aumentar o peso?

8. How often do you think about changing the amount of food supplements you use to put on weight?

8. Com que frequência você pensa em mudar a quantidade de suplementos alimentares que você usa para aumentar o peso?

9. How often do you worry about changing the amount of 9. Com que frequência você se preocupa em mudar a dieting supplements you use to gain weight? quantidade de suplementos alimentares que você usa para aumentar o peso?

\section{Strategies for Body Change to Increase Muscle Tone}

\section{Estratégias de mudança corporal para Aumento do Tônus Muscular}

1. How often do you worry about changing your eating to improve your muscle tone?

2. How often do you change your eating to improve your muscle tone?

3. How often do you think about changing your eating to improve your muscle tone?

4. How often do you think about exercising more to improve your muscle tone?

5. How often do you exercise more to improve your muscle tone?

6. How often do you worry about exercising more to improve your muscle tone?

\section{Strategies for Body Change to Increase Muscle Size}

1. How often do you worry about changing your eating to get bigger muscles?

2. How often do you change your eating to get bigger muscles?

3. How often do you think about changing your eating to get bigger muscles?

4. How often do you think about exercising more to get bigger muscles?

5. How often do you exercise more to get bigger muscles?

6. How often do you worry about exercising more to get bigger muscles?
1. Com que frequência você se preocupa em mudar sua alimentação para aumentar seu tônus muscular?

2. Com que frequência você muda sua alimentação para aumentar seu tônus muscular?

3. Com que frequência você pensa em mudar sua alimentação para aumentar seu tônus muscular?

4. Com que frequência você pensa em fazer mais exercícios para aumentar seu tônus muscular?

5. Com que frequência você faz mais exercícios para aumentar seu tônus muscular?

6. Com que frequência você se preocupa em fazer mais exercícios para aumentar seu tônus muscular?

\section{Estratégias de mudança corporal para Aumento do Tamanho Muscular}

1. Com que frequência você se preocupa em mudar sua alimentação para tornar seus músculos maiores? 2. Com que frequência você muda sua alimentação para tornar seus músculos maiores?

3. Com que frequência você pensa em mudar sua alimentação para tornar seus músculos maiores?

4. Com que frequência você pensa em fazer mais exercícios para tornar seus músculos maiores?

5. Com que frequência você faz mais exercícios para tornar seus músculos maiores?

6. Com que frequência você se preocupa em fazer mais exercícios para tornar seus músculos maiores? 
Quadro 2. Questionário de Mudança Corporal.

Este questionário foi preparado para obter informações de como você se sente em relação ao seu corpo e de suas atitudes para mudá-lo. Suas respostas são completamente anônimas. Ninguém saberá suas respostas.

Não há respostas certas ou erradas. Nós apenas queremos saber como você se sente e o que você faz. É importante que não leve muito tempo para responder cada questão. Simplesmente marque a resposta que melhor se aplicar a você.

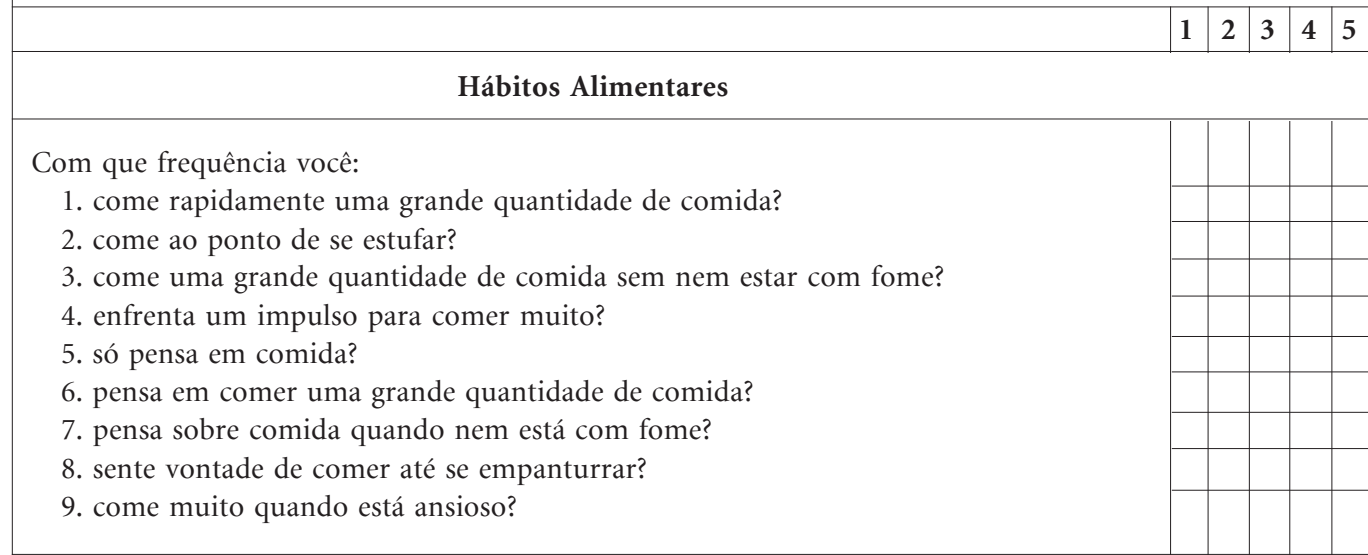

Suplementos Alimentares

Com que frequência você:

1. usa substâncias para perder peso, por exemplo, remédios para emagrecer?

2. sente vontade de usar substâncias para perder peso, por exemplo, remédios para emagrecer?

3. pensa em usar substâncias para perder peso, por exemplo, remédios para emagrecer? 4. preocupa-se em usar substâncias para perder peso, por exemplo, remédios para emagrecer?

5. usa suplementos alimentares (por exemplo, sustagen) para aumentar o tamanho de seus músculos?

6. sente vontade de tomar suplementos alimentares (por exemplo, sustagen) para aumentar o tamanho do seus músculos?

7. pensa em tomar suplementos alimentares (por exemplo, sustagen) para aumentar o tamanho de seus músculos?

8. usa esteroides-anabolizantes?

9. pensa em usar esteroides-anabolizantes?

\section{Estratégias de Mudança Corporal para perder peso}

Com que frequência você:

1. pensa em comer menos para perder peso?

2. come menos para perder peso?

3. preocupa-se em comer menos para perder peso?

4. pensa em fazer mais exercícios para perder peso?

5. faz mais exercícios para perder peso?

6. preocupa-se em fazer mais exercícios para perder peso?

7. muda a quantidade de suplementos alimentares que você usa para perder peso?

8. pensa em mudar a quantidade de suplementos alimentares que você usa para perder peso?

9. preocupa-se em mudar a quantidade de suplementos alimentares que você usa para perder peso?
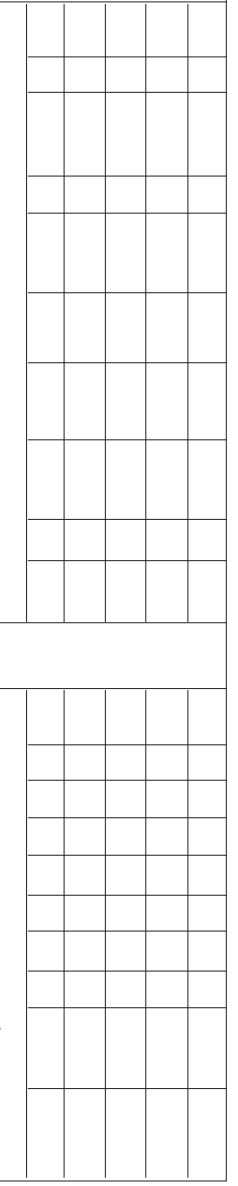

continua 
Quadro 1. continuação

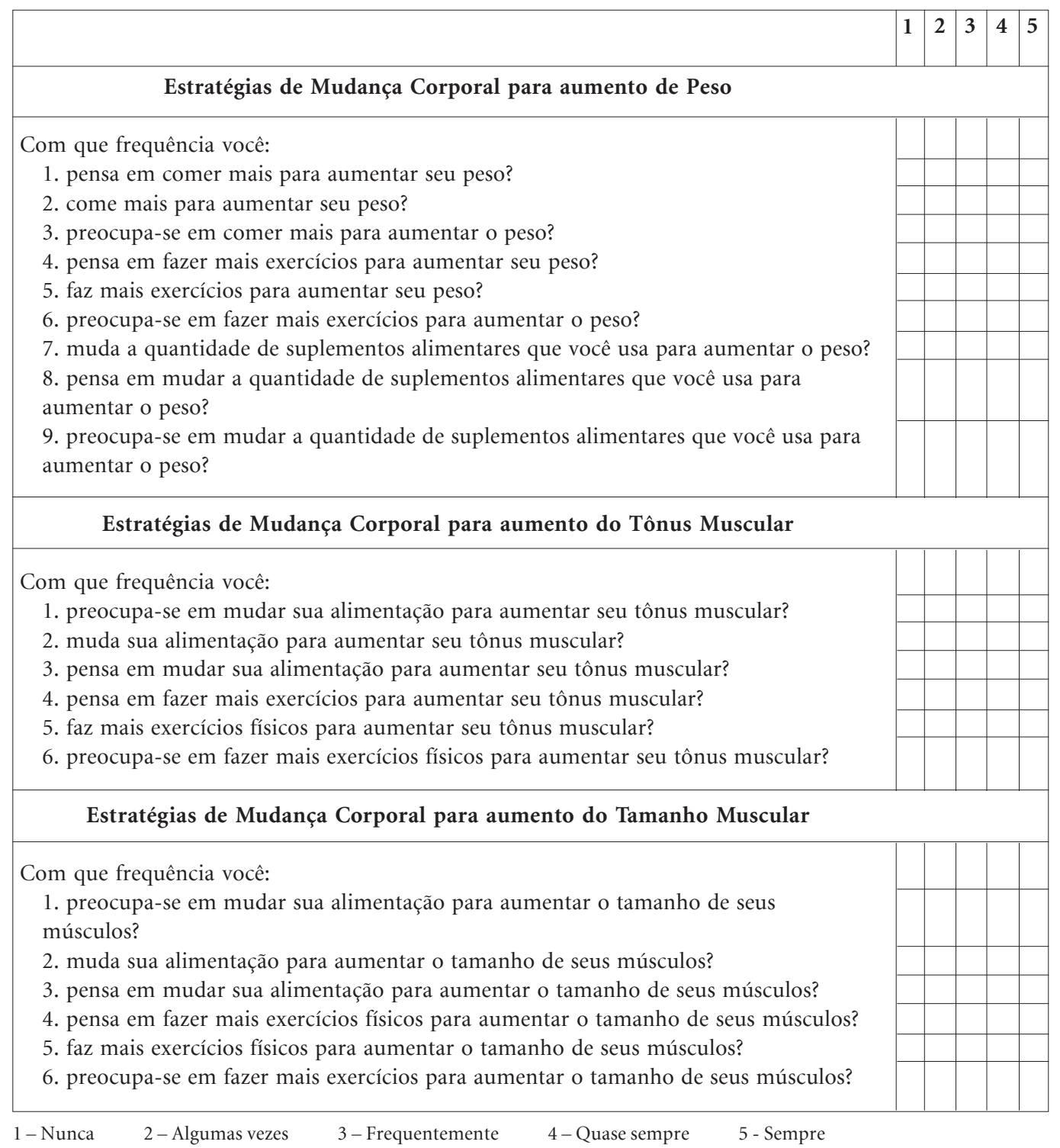

Duas alterações foram propostas na forma de apresentação. A primeira transformou a expressão "Com que frequência", presente em todas as questões, em uma única chamada para cada subescala. Desta forma, cada item iniciouse com o tema a ser avaliado. A segunda alteração referiu-se à apresentação das opções de respostas. No instrumento original, logo abaixo de cada item há 5 opções padronizadas por palavras, de "nunca" a "sempre". Para a nossa versão associou-se cada opção a um número, logo após o enunciado de apresentação do instrumento. Assim, ao lado de cada questão são apresentadas 5 escolhas numéricas, e o preenchimento con- siste na opção de uma única resposta. Com estas adaptações garantiu-se uma maior objetividade e clareza do instrumento, facilitando sua leitura e o seu preenchimento.

Vale observar que para cada subescala, alguns temas são avaliados somente com a distinção dos construtos afetivos (sente), cognitivo (pensa e preocupa-se) e comportamental (usa, faz, come, muda). Esta característica se repete ao longo do questionário. Para melhor elucidar esse ponto, tomamos como exemplo a questão do comportamento alimentar explorada na subescala "Estratégias de mudança corporal para aumento de peso", que avalia o pensamento, o sen- 
timento e a preocupação em relação ao ato de comer. Apesar de se parecerem, as questões avaliam construtos distintos para o ato de comer. Neste sentido são pontuais as observações realizadas pela população alvo, que identificou certa repetição das questões, o que de certa maneira é justificado pela lógica estrutural do instrumento. No entanto, estudos relacionados a fatores de risco associados à imagem corporal comprovam a importância da distinção destes construtos (afetivo, cognitivo e comportamental) ${ }^{14}$. Ademais, como pontuam as autoras do QMC, cada subescala poderá ser aplicada independentemente das demais que compõem o instrumento. Estes argumentos justificam a manutenção das questões, como seus respectivos desdobramentos, conforme proposto no instrumento original.

É necessário destacar, entretanto, que a avaliação da equivalência semântica, realizada no presente estudo, não garante a aplicabilidade do instrumento, mas representa o primeiro passo para que uma escala de qualidade esteja disponível para aplicação em amostras brasileiras. Este estudo se propôs a descrever minuciosamente o processo de avaliação da equivalência semântica de um instrumento traduzido, a exemplo de outros estudos que se preocuparam com a qualidade do processo de adaptação transcultural ${ }^{20,21,25,26}$.

Em relação à compreensão verbal do instrumento, tanto especialistas quanto estudantes relataram compreensão suficiente das questões que compõem a escala, apresentado valores médios superiores a 3, como proposto por Conti et al. ${ }^{21}$. A versão final do instrumento foi elaborada com base nas sugestões dos participantes, como indi- cado por Beaton et al. ${ }^{27}$, os quais propõem que a tradução de um instrumento apenas poderá ser finalizada após a avaliação do mesmo por uma amostra da população-alvo.

Cabe ainda salientar que os transtornos alimentares e os distúrbios da imagem corporal estão cada vez mais presentes na população brasileira. Estudos recentes, como o de Martins et al. ${ }^{28}$, encontraram valores preocupantes de prevalência de sintomas de transtornos alimentares entre a população adolescente, superando os verificados em outros estudos nacionais e internacionais. Estes resultados reafirmam a necessidade de instrumentos válidos destinados a avaliar os fatores de risco para o desenvolvimento destes transtornos.

De acordo com Reichenheim e Moraes ${ }^{18}$ é de extrema importância para os estudos epidemiológicos zelar pela qualidade da informação, visto ser este um elo entre o conteúdo teórico e o dado que expressa a realidade empírica. Sendo assim, o rigor dispensado no processo de aferição deve ocupar um lugar de destaque nesta prática. Este cuidado foi tomado na presente pesquisa.

O Questionário de Mudança Corporal (QMC) encontra-se pronto para novos estudos que possam avaliar suas condições psicométricas. O processo de tradução e avaliação da equivalência semântica é apenas o primeiro passo para que um instrumento possa ser utilizado em outro contexto cultural. Sendo assim, são ainda necessárias análises de sua consistência interna, validade externa, equivalência de mensuração e reprodutibilidade para que o QMC seja considerado apto para uso em amostras brasileiras.

\section{Colaboradores}

MA Conti, MEC Ferreira, ACS Amaral, N Hearst, TA Cordás e FB Scagliusi participaram igualmente de todas as etapas de elaboração do artigo. 


\section{Referências}

1. Ricciardelli L, McCabe MP. Psychometric evaluation of the Body Change Inventory: An assessment instrument for adolescent boys and girls. Eat Behav 2002; 3(1):45-59.

2. McCabe MP, Ricciardelli L, Waqa G, Goundar R, Fotu K. Body Image and body change strategies among adolescent males and females from Fiji, Tonga and Austrália. Body Image 2009; 6(4):299-303.

3. McCabe MP, Ricciardelli L, Holt K. Are there different sociocultural influences on body image and body change strategies for overweight adolescent boys and girls? Eat Behav 2010; 11(3):156-163.

4. McCabe MP, Ricciardelli LA, Banfield S. Body Image, strategies to change muscles and weight, and puberty. Do they impact on positive and negative affect among adolescent boys and girls? Eat Behav 2001; 2(2):129-149.

5. Cafri G, Yamamyia Y, Brannick M, Thompson JK. The influence of Sociocultural Factors on Body Image: a meta-analysis. Clin Psychol Sci Pract 2005; 12(4):421-433.

6. Ricciardelli LA, McCabe MP, Holt KE, Finemore J. A biopsychosocial model for understanding body image and body change strategies among children. Applied Developmental Psychology 2003; 24(4):475-485.

7. McCabe MP, Ricciardelli LA. Body Image and Body Change Techniques among young adolescent boys. Eur Eat Disorders Rev 2001; 9(5):335-347.

8. McCabe MP, Vincent MA. Development of the Body Modification and Excessive Exercise Scales for Adolescents. Assessment 2002; 9(2):131-141.

9. Iriart JAB, Chaves JC, Orleans RG. Culto ao corpo e uso de anabolizantes entre praticantes de musculação. Cad Saude Publica 2009; 25(4):773-782.

10. Nunes MA, Barros FC, Olinto MTA, Camey S, Mari JD. Prevalence of abnormal eating behaviours and inappropriate methods of weight control in young women from Brazil: a population-based study. Eat Weight Disord 2003; 8(2):100-106.

11. Mellor D, McCabe M, Ricciardelli L, Merino ME. Body Dissatisfaction and body change behaviors in Chile: The role of sociocultural factors. Body Image 2008; 5(2):205-215.

12. Xua X, Mellor D, Kiehne M, Ricciardelli L, McCabe MP, Xu Y. Body Dissatisfaction, engagement in body change behaviors and sociocultural influences on body image among Chinese adolescents. Body Image 2010; 7(2):156-164.

13. Mellor D, McCabe M, Ricciardelli L, Yeow J, Daliza N, Fizlee N, Hapidzal M. Sociocultural influences on body dissatisfaction and body change behaviors among Malaysian adolescents. Body Image 2009; 6(2):121-128.

14. Thompson JK. The (mis) measurement of body image: ten strategies to improve assessment for applied and research purposes. Body Image 2004; 1(1):7-14.

15. McCabe MP, Ricciardelli L. A longitudinal study of pubertal timing and extreme Body Change Behaviors among adolescent boys and girls. Adolescence 2004; 39(153):145-166.

16. McCabe MP, Ricciardelli L. Sociocultural Influences on Body Image and Body Changes among adolescent boys and girls. J Soc Psychol 2003; 143(1):5-26.
17. McCabe MP, Ricciardelli L, Finemore J. The role of puberty, media and popularity with peers on strategies to increase weight, decrease weight and increase muscle tone among adolescent boys and girls. J Psychosom Res 2002; 52(3):145-153.

18. Reichenheim ME, Moraes CL. Operacionalização de adaptação transcultural de instrumentos de aferição usados em epidemiologia. Rev Saude Publica 2007; 41(4):665-673.

19. Conti MA, Latorre MRDO, Slater B. Tradução, validade e reprodutibilidade da EEICA - Escala de Evaluación da Insatisfación Corporal para Adolescentes - no Brasil. Rev Saude Publica 2009; 43(3):515-524.

20. Amaral ACS, Cordás TA, Conti MA, Ferreira MEC. Equivalência Semântica e avaliação da consistência interna da versão em português do Sociocultural Attitudes Towards Appearance Questionnaire-3 (SATAQ-3). Cad Saude Publica 2011; 27(8):1487-1497.

21. Conti MA, Scagliusi F, Queiroz GKO, Hearst N, Cordas TA. Adaptação transcultural: tradução e validação de conteúdo para o idioma português do modelo da Tripartite Influence Scale de insatisfação corporal. Cad Saude Publica 2010; 26(3):503-513.

22. Brasil. Ministério da Saúde. Conselho Nacional de Saúde. Resolução no ${ }^{\circ}$. 196/1996. Diretrizes e Normas Regulamentadoras de Pesquisas Envolvendo Seres Humanos. Diário Oficial da União 1996; out 10.

23. van Widenfelt BM, Trefers PDA, Beurs E, Siebelink BM, Koudijs E. Translation and Cross-cultural adaptation of assessment instruments used in psychological research with children and families. Clin Child Fam Psychol Rev 2005; 8(2):135-147.

24. Jorge MR. Adaptação transcultural de instrumentos de pesquisa em saúde mental. In: Gorestein C, Andrade LHSG, Zuardi AW, organizadores. Escalas de avaliação clínica em psiquiatria e psicofarmacologia. São Paulo: Lemos Editorial; 2000. p. 53-59.

25. Holanda WTG, Lima MLC, Figueroa JN. Adaptação transcultural de um instrumento de avaliação do handicap auditivo para portadores de perda auditiva induzida pelo ruído ocupacional. Cien Saude Colet 2011; 16(Supl. 1):755-767.

26. Franca C, Colares V. Validação do National College Health Risk Behavior Survey para utilização com universitários brasileiros. Cien Saude Colet 2010; 15(Supl. 1):1209-1215.

27. Beaton DE, Bombardier C, Guillemin F, Ferraz MB. Guidelines for the process of Cross-Cultural Adaptation of Self-Report Measures. Spine 2000; 25(24): 3186-3191.

28. Martins CR, Pelegrini A, Matheus SC, Petroski EL. Insatisfação com a imagem corporal e relação com estado nutricional, adiposidade corporal e sintomas de anorexia e bulimia em adolescentes. Rev Psiquiatr RS 2010; 32(1):19-23.

Artigo apresentado em 06/05/2011

Aprovado em 14/09/2011 